\title{
Intersección entre la evidencialidad y la atenuación: el pretérito imperfecto evidencial y el futuro narrativo
}

\author{
INTERSECTIONS BETWEEN EVIDENTIALITY AND ATTENUATION: THE EVIDENTIAL \\ IMPERFECT AND NARRATIVE FUTURE
}

\author{
Gerda HABLER \\ Universidad de Potsdam
}

Resumen

Además de los recursos léxicos que se encuentran parcialmente de camino a la pragmaticalización, la atenuación, como un fenómeno pragmáticodiscursivo, puede realizarse mediante formas verbales del indicativo, las mismas que pueden ser utilizadas sin su valor temporal prototípico. El pretérito imperfecto, por ejemplo, asume la función de un marcador evidencial, sobre todo, en textos narrativos y periodísticos, para indicar que la información procede de una tercera fuente. De esta manera, el locutor se distancia de sus enunciados y realiza simultáneamente una atenuación del contenido proposicional. Esta función del pretérito imperfecto se explica por su aspectualidad imperfectiva, que conduce a la modalidad debido a su indeterminación semántica. Es más infrecuente, en español, el uso del futuro narrativo que expresa situaciones pasadas con respecto al momento de habla, pero que también hace referencia a situaciones venideras desde un momento en el pasado, las que son presupuestas por el locutor. En esta contribución, se estudiará el valor evidencial del pretérito imperfecto y del futuro, así como el papel que desempeñan en la atenuación.

\footnotetext{
Palabras clave: pretérito imperfecto, futuro narrativo, aspectualidad, atenuación, evidencialidad, responsabilidad del hablante
}

Abstract:

In addition to lexical means which are partially on the way to pragmaticalization, attenuation as a pragmatic discourse phenomenon can be expressed by verbal forms of the indicative mood which can be used without their prototypical temporal value. For example, the imperfect past tense assumes the function of an evidential marker, especially in narrative and journalistic texts, and indicates that the information comes from a secondary source. By using the imperfect past tense the speaker distances themself from his utterance and at the same time he provides an attenuation of its propositional content. This function of the past imperfect tense can be explained by imperfective aspectuality, which leads to modality due to its semantic indeterminacy. In Spanish the narrative future is less frequent. This use of the future designates situations in the past and it takes into account the speech moment, but also future situations seen from a moment in the past, adopted by the speaker. The evidential value of the Spanish imperfect past and future tense, as well as their function in attenuation will be studied in this contribution.

KEY WORDS: imperfect past tense, narrative future, aspectuality, attenuation, evidentiality, speaker's stance 


\section{normas}

Intersección entre la evidencialidad y la atenuación: el pretérito imperfecto evidencial y el futuro narrativo | Gerda Haßler

La atenuación, como un fenómeno pragmático-discursivo. puede realizarse mediante formas verbales del indicativo, las mismas que pueden ser usadas sin su valor temporal prototípico y que podrían encontrarse en un proceso de pragmaticalización. En esta contribución no se estudiarán los marcadores léxicos ${ }^{1}$ de atenuación ni tampoco el subjuntivo como modo de la irrealidad, sino que el enfoque será puesto en dos formas verbales del indicativo que son susceptibles de mitigar el contenido proposicional en contextos narrativos, es decir, el pretérito imperfecto y el futuro.

\section{EFECTOS DE ATENUACIÓN QUE PUEDEN REALIZARSE MEDIANTE EL PRETÉRITO IMPERFECTO Y EL FUTURO SIMPLE}

El concepto de atenuación tomado para esta contribución implica mitigaciones del contenido proposicional que no son realizados por elementos atenuadores especializados en esta función. Además, se toma en cuenta un concepto que puede conciliar diferentes modos de entender la atenuación. Para ello, se utiliza el postulado de Antonio Briz y Marta Albelda (2013: 292) que consideran que "la atenuación es una actividad argumentativa (retórica) estratégica de minimización de la fuerza ilocutiva y del papel de los participantes en la enunciación para lograr llegar con éxito a la meta prevista, y que es utilizada en contextos situacionales de menos inmediatez o que requieren o se desea presenten menos inmediatez comunicativa». En contextos narrativos, la ilocución consiste primordialmente en la mención y la afirmación de acontecimientos, la fuerza ilocutiva se reduce, restringiendo la validez de la proposición o de su relación con el escritor. Cuando el escritor de un texto narrativo o periodístico mitiga su papel como autor o fuente de la información, presenta el enunciado como atenuado y con menos inmediatez comunicativa.

Cuando «dicha estrategia consiste lingüísticamente en minorar, minimizar, mitigar, debilitar la acción e intención o el efecto que estas puedan tener o haber tenido en la interacción» (Briz/Abeldia 2013: 293; cf. también Briz 1995, 1998), es posible considerar también como atenuación la minoración y el distanciamiento del mensaje mediante el uso de ciertas formas verbales. El uso de estas formas verbales debilita la fuerza ilocutiva, de tal manera que el hablante logra así distanciarse de su para crear un efecto amable y cortés frente a sus interlocutores. Mediante el uso del pretérito imperfecto se reduce la fuerza ilocutiva en oraciones, como la siguiente, cuando una persona entra en una tienda y se dirige así al vendedor:

(1) Quería una barra de pan.

En este caso se trata del imperfecto de cortesía que minimiza la fuerza ilocutiva de un requerimiento o pedido, por medio del cual el hablante (comprador) se expresa cortésmente hacia su interlocutor (vendedor). Esta forma de suavizar o mitigar la expresión resulta muy frecuente en la lengua hablada, sobre todo, cuando queremos pedir algo. Al no imponernos o centrarnos solo en nuestro deseo, formulamos nuestra petición de modo indirecto. De este modo, no solo otorgamos al interlocutor una mayor margen para rechazar o no nuestra petición, sino que el interlocutor es tratado con estima y consideración por medio del hablante, cuya imagen se intepreta como cortés y agradable:

\footnotetext{
${ }^{1}$ Los marcadores léxicos han sido estudiados en Haßler (2015) y Böhm/Haßler/Hennemann (2017).
} 
"[...] the addressee is treated with consideration, the adressor is improving his chances of being considered likeable» (Beeching 2002: 21). El efecto de cortesía-atenuación se logra mediante el uso del pretérito imperfecto - como lo hemos visto en el ejemplo anterior (1)el cual es usado sin su valor temporal prototípico del pasado, ya que es usado en vez del presente (cf. Quería una barra de pan vs. Quiero una barra de pan). En los siguientes ejemplos, el uso del pretérito imperfecto atenúa la fuerza ilocutiva de los enunciados:

(2) ¿Tienes un momento para hablar? Tenía que pedirte una cosa.

(3) Perdone, estaba buscando un libro sobre el fútbol.

El uso del pretérito imperfecto de cortesía se encuentra también en todas las lenguas románicas, tal y como lo muestran los siguientes ejemplos:

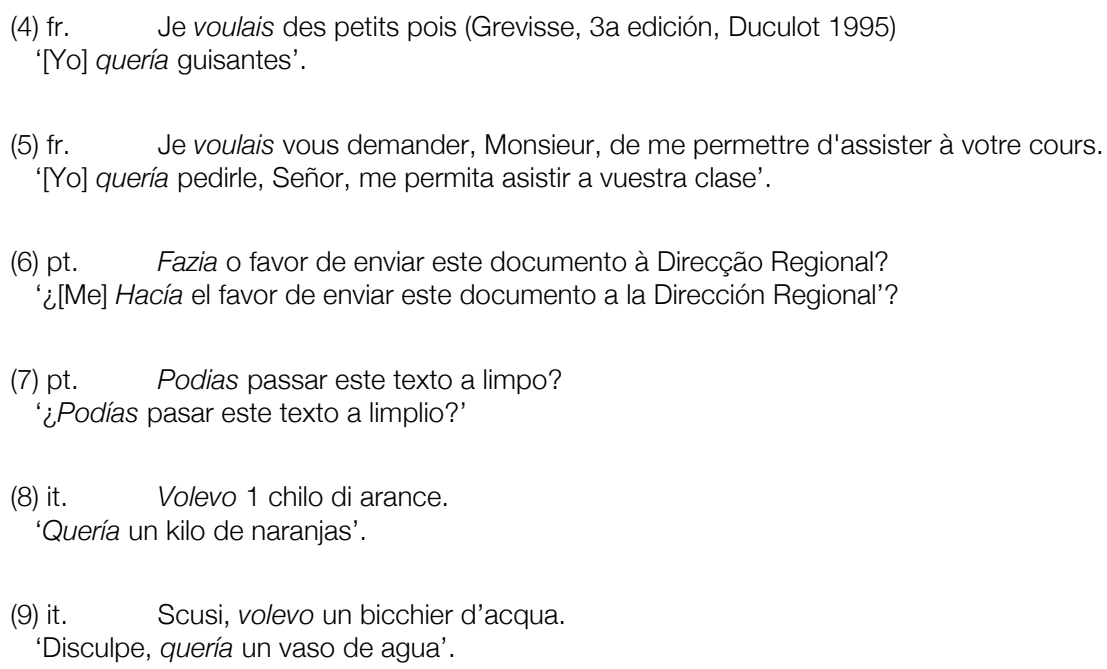

Además de esta función atenuativa en el contexto de la cortesía, el pretérito imperfecto puede mitigar el grado de compromiso o responsabilidad que tiene el hablante con respecto al valor de verdad de la situación expresada en su enunciado. Esta mitigación del grado de compromiso o responsabilidad del hablante para asumir la veracidad de su proposición puede encontrarse, por lo general, en el contexto narrativo-periodístico (cf. Böhm 2013: 125; Böhm/Hennemann 2014: 198; Böhm 2016). Según algunos autores, como Reyes (1990: 29), se trata del pretérito imperfecto con «valor citativo» u «oblicuo», por medio del cual se restringe el valor de verdad de una afirmación, lo que atenúa su contenido proposicional. En su ejemplo «Hoy daba una conferencia María», Reyes (1990: 17) señala que el uso del pretérito imperfecto equivale a decir tal fuente informó (Reyes 1990: 32), por medio del cual se trata de deslindar responsabilidades: «el hablante no asume enteramente lo que dice, y para eso indica (por ejemplo, usando el imperfecto de ese modo peculiar) que su conocimiento procede del testimonio de otro" (Reyes 1994: 12; cf. Böhm 2016: 26). ${ }^{2}$ De este modo, mediante este efecto de atenuación motivado por el pretérito imperfecto el hablante se distancia de sus enunciados, indicando implícitamente que él no es el autor de la información, por lo que se logra crear el efecto pragmático de

\footnotetext{
${ }^{2}$ Para el uso citativo del pretérito imperfecto, cf. también Leonetti/Escandell (2003).
} 


\section{normas}

Intersección entre la evidencialidad y la atenuación: el pretérito imperfecto evidencial y el futuro narrativo | Gerda Haßler

reserva y discreción por parte del hablante, ya que no quiere comprometerse con lo que dice. El uso del pretérito imperfecto con esta función atenuadora de la responsabilidad del hablante y el distanciamiento de sus enunciados aparece, sobre todo, en español.

El futuro simple también puede usarse con efectos de atenuación, sobre todo, en contextos de cortesía, para suavizar una petición, como en «Usted me dirá, lo que tengo que hacer» en vez de "Dígame, lo que tengo que hacer». En otros contextos, el uso del futuro simple también puede mitigar el grado de certeza sobre un contenido proposicional, como por ejemplo, "Serán las tres". En este caso, el hablante no solo reduce el valor de verdad sobre el contenido de su enunciado, sino que expresa al mismo tiempo incertidumbre y probabilidad. Más adelante, se tratarán otros casos de atenuación motivados por el futuro, sobre todo, como futuro narrativo en relación con la evidencialidad.

\section{EL PRETÉRITO IMPERFECTO COMO MARCADOR DE EVIDENCIALIDAD Y DE ATENUACIÓN}

La indicación de la procedencia de una información o de una fuente o de la experiencia o reflexión del hablante puede intensificar o atenuar el contenido de la proposición. Mientras que las construcciones evidenciales pueden dar más fuerza al contenido proposicional, subrayando la inmediatez de la experiencia o la autoridad de la fuente, ${ }^{3}$ el pretérito imperfecto implica, en algunos contextos, una cierta atenuación. Así, como ya se ha mencionado anteriormente, el pretérito imperfecto puede ser usado sin su valor temporal prototípico (forma verbal relativa que depende de otro pretérito o de un adjunto temporal para establecer su referencia temporal). En el siguiente ejemplo (10), el pretérito imperfecto no solo aparece como el miembro principal sintáctico en la proposición (es decir, como una forma verbal absoluta), sino que describe una situación delimitada, por lo que su significado aspectual imperfectivo queda escindido:

\footnotetext{
(10) Peres reconocía ayer que el presidente sirio, Hafez El Assad, será el gran ausente de la cumbre, si bien ha sido informado por Mubarak de todos los detalles. (La Vanguardia, 02/02/1995, Política)
}

En el enunciado anterior, se relata una acción única, temporalmente localizada y terminada, lo que sugeriría el uso del pretérito perfecto simple, tal y como lo encontramos efectivamente en un contexto comparable:

\section{(11) Juppé reconoció ayer que los ingresos fiscales «están en una auténtica situación de siniestro", al no haberse cumplido las previsiones de crecimiento. (La Vanguardia, 30/09/1995, Economía y Hacienda)}

En el ejemplo (11) se constata simplemente un hecho, el aspecto perfectivo expresado por reconoció presenta la acción como concluida y en su totalidad. El uso del pretérito imperfecto en textos periodísticos, como en el ejemplo 10, reduce y mitiga el grado de compromiso o responsabilidad del periodista frente a su información y atenúa la fuerza ilocutiva de la afirmación, ya que la forma imperfectiva no presenta la situación como delimitada (cf. Dessì Schmid 2014; Böhm 2016). Al mismo tiempo, se caracteriza sutilmente la información comunicada como obtenida de tercera mano. Entonces, la mitigación de la responsibilidad y del compromiso del hablante, así como la atenuación del contenido proposicional están relacionadas con la referencia implícita a una fuente de

\footnotetext{
${ }^{3}$ Para los valores de los evidenciales, cf. por ejemplo, Squartini (2001), Haßler (2015), Haßler (2016: 352-379).
} 


\title{
normas
}

Intersección entre la evidencialidad y la atenuación: el pretérito imperfecto evidencial y el futuro narrativo | Gerda Haßler

información externa (obtenida de tercera mano). Por lo tanto, se puede establecer una conexión semántico-pragmática entre la atenuación y la evidencialidad ${ }^{4}$ indirecta.

Aunque este uso del pretérito imperfecto ha sido relacionado con su función citativa y solo dentro del marco de sus valores pragmáticos y discursivos en el discurso oral (Reyes 1990, Gutiérrez Aráus 1995) y no tanto en el escrito, el enfoque de estudio permite obtener otra perspectiva del uso del pretérito imperfecto en términos de una intersección semántica entre la atenuación y la evidencialidad indirecta, la cual no ha sido tratada, de esa forma, hasta ahora. Entre algunos autores que han estudiado el uso del pretérito imperfecto en discursos escritos, se encuentran, por ejemplo, García Fernández (2004) que se refiere al uso del pretérito imperfecto como imperfecto narrativo en textos periodísticos y Díaz Salgado (2000) que relaciona este uso en textos periodísticos más bien con una función pragmática y de estrategia comunicativa. También Verónica Böhm (2016), en su tesis sobre la imperfectividad en la prensa española y su relación con las categorías semánticas de modalidad y evidencialidad, ha estudiado exhaustivamente los usos del pretérito imperfecto en cuanto a sus funciones primarias o prototípicas, relacionadas con su valor temporal relativo y aspectual imperfectivo, y sus funciones secundarias o no prototípicas relacionadas con su valor modal epistémico y evidencial indirecto.

Por lo general, el pretérito imperfecto no solo enfatiza la duración o el transcurso de una situación, sino también motiva el significado de iteración, continuidad o repetición (ejemplo 12), así como de hábito (ejemplo 13) de una acción, lo que está relacionado con su carácter aspectual imperfectivo (cf. Böhm 2016: 21): (12) Su padre, Webster Todd, fue un contratista millonario que construía edificios para los
Rockefeller. [...] (CREA, El Mundo, Política, 31/03/1995)

(13) Asunción se levantaba a las 6,30 de la mañana; a partir de las 7 leía la Prensa, y a las 8,30, fresco como una rosa, estaba listo para recibir la llamada diaria de Belloch [...] (CREA, El Mundo, Gobierno, 15/01/1996)

Asimismo, su carácter temporal relativo requiere de otros elementos gramaticales para su contextualización, como por ejemplo, cuando aparece como 'copretérito' de otro pretérito (el pretérito perfecto simple) para expresar simultaneidad de una acción en el pasado:

\begin{abstract}
(14) El brasileño no conocía muchas cosas de la Real cuando llegó a San Sebastián. Ahora, después de dos partidos, ya se puede hacer una idea. «La Real tiene grandes jugadores, hombres con muy buenas condiciones [...]” (CREA, El Diario Vasco, Deportes, 31/01/2001)
\end{abstract}

No obstante, en los siguientes ejemplos (15) y (16) no se interpreta el uso del pretérito imperfecto como «una forma verbal relativa que depende de otra situación del pasado» (Böhm 2016: 22). El pretérito imperfecto es usado en vez del pretérito perfecto simple como una forma verbal absoluta para expresar situaciones puntuales y únicas que tuvieron lugar en el pasado. Para comparar, véanse los ejemplos contrarios (17) y (18), donde aparece el pretérito perfecto simple en el mismo contexto, cuyo uso es, por lo general, el más normal y gramaticalmente el más adecuado ${ }^{5}$ :

\footnotetext{
${ }_{5}^{4}$ Sobre la evidencialidad, cf. Cornillie (2007), Hennemann (2013), Diewald (1999), Diewald/Smirnova (2010).

5 De acuerdo con la Nueva Gramática de la lengua española (RAE 2009), «el pretérito perfecto simple es compatible con los predicados télicos porque estos contienen un rasgo interno de delimitación: Se asomó por la ventana (es decir, 'en cierto
} 


\section{normas}

Intersección entre la evidencialidad y la atenuación: el pretérito imperfecto evidencial y el futuro narrativo | Gerda Haßler

(15) El Papa Francisco recibía el lunes en el Palacio Apostólico a la última tanda de prelados de las provincias eclesiásticas del sur de España, incluidas las Islas Canarias, Ceuta y Melilla. (GlossaNet, $A B C, 13 / 03 / 2014)$

(16) Wall Street abrió hoy sin tendencia definida y el Dow Jones de Industriales bajaba un $0,07 \%$, con los mercados cautelosos a la espera del inicio de la temporada de resultados trimestrales. (GlossaNet, El País, 10/04/2014)

(17) Este lunes, Bachelet recibió a los presidentes de los partidos de la Nueva Mayoría, que la felicitaron por el triunfo y manifestaron su compromiso para trabajar en su programa de gobierno. (GlossaNet, El Mundo, 19/12/2013)

(18) Así, a nivel nacional, la compraventa de viviendas bajó un 15,9\% el pasado mes de noviembre respecto al mismo mes de 2012, hasta un total de 21.847 operaciones, registrando así la segunda cifra más baja del año tras la de marzo (22.100 transacciones) y una de las menores de toda la serie, iniciada en 2007. (GlossaNet, El Mundo, 16/01/2014)

De este modo, en los ejemplos (15) y (16), el pretérito imperfecto ya no expresa más sus significados primarios o funciones prototíicicas, sino (otros) significados secundarios que están relacionados con la actitud epistémica del hablante frente a su enunciado y a la fuente de información, lo que se puede subsumir bajo la noción de evidencialidad indirecta (Böhm 2016: 21).

Este significado secundario del pretérito imperfecto puede derivarse de su aspectualidad imperfectiva, por medio de la cual se contempla una acción en su transcurso y desarrollo. Mientras que no se consideren el inicio y el final de una acción, y la acción o situación sea vista como indeterminada e inconclusa, la validez de la proposición se presenta también como abierta o indeterminada ${ }^{6}$. De manera que la aspectualidad imperfectiva conduce a la modalidad debido a su indeterminación (Haßler 2012: 141). Este puente metonímico puede representarse, según Böhm (2016: 321), de la siguiente manera:

momento')» (RAE 2009: 1763). Así, los verbos de logro, como recibir y bajar compatibilizan mucho mejor con el pretérito perfecto simple.

${ }^{6}$ Sobre la definición de la noción de 'aspect', cf. Dessí Schmid (2016). 


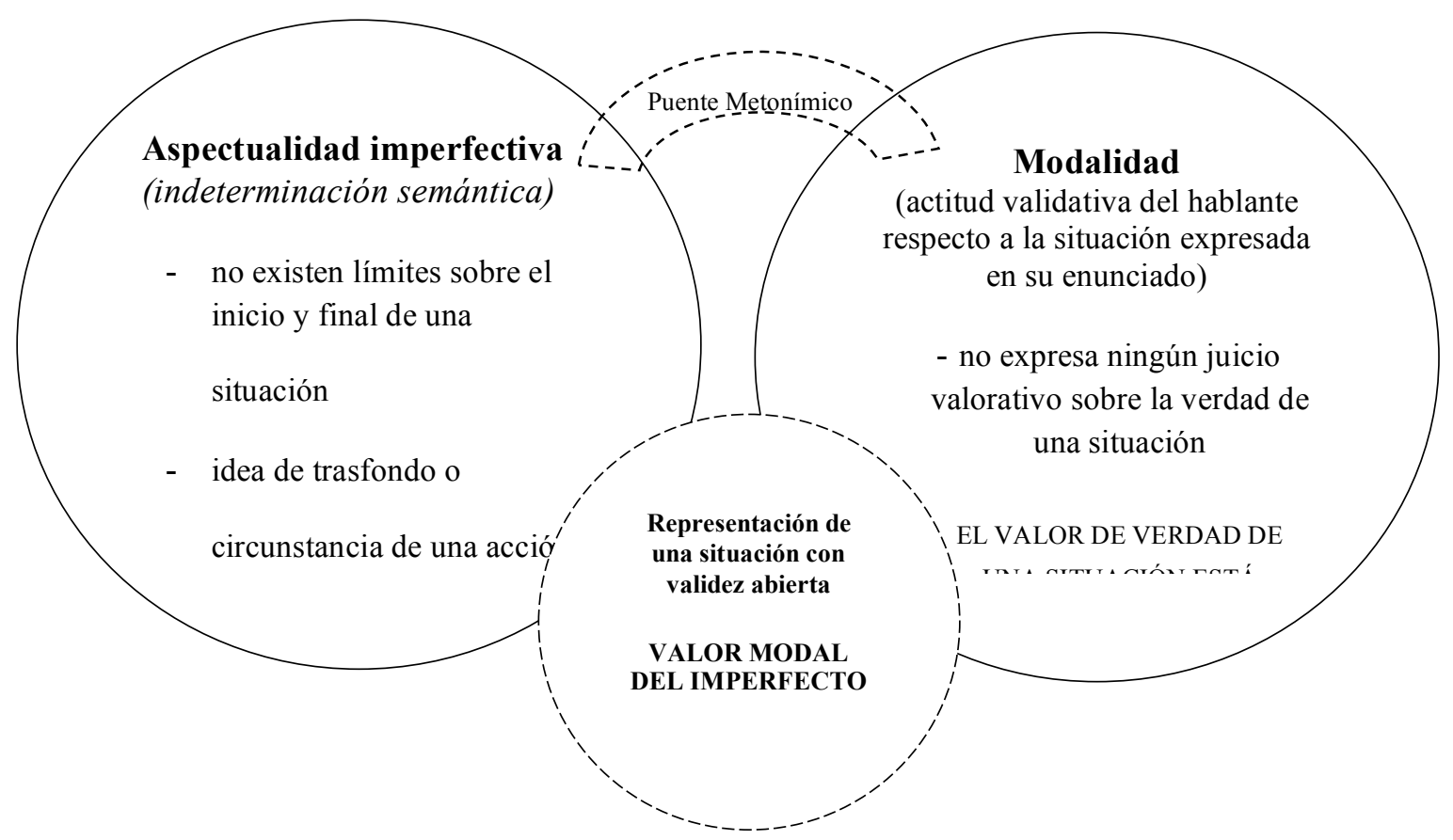

Utilizando el pretérito imperfecto, el hablante toma una decisión muy económica, ligando dos dominios cognitivos, el de la no-limitación de una situación (aspectualidad imperfectiva) y el de la validez abierta (modalidad). El saber enciclopédico del hablante con respecto a que una situación no está determinada en cuanto a su inicio y final tiene un grado menor de asertividad y posibilita la transposición a la modalización de una proposición. Así, en el siguiente enunciado, el pretérito imperfecto sustituye al condicional en la construcción de irrealidad:

(19) Si hubiese otra taberna abierta a estas horas, te podías despedir de mí como cliente. (I. Aldecoa, En el kilómetro 400, en Libro de Lecturas, 37.cf. Fernández Ramírez 1986: 276)

En algunos tipos de textos, sobre todo, en el lenguaje periodístico, el grado de compromiso y la responsabilidad del hablante es muy importante, por lo que la atenuación podría ser considerada como una estrategia pragmática-periodística para reducir o mitigar la responsabilidad del periodista con respecto a lo que está informando y distanciarse, de ese modo, de sus enunciados. Y precisamente, mediante el uso secundario del pretérito imperfecto, como ya lo hemos mencionado anteriormente y hemos visto en ejemplos anteriores, se logra crear este efecto de atenuación de la responsabilidad y compromiso del hablante frente a lo que dice, lo que está naturalmente conectado con la evidencialidad. A continuación, véanse más ejemplos (20-22), donde las formas del pretérito imperfecto describen acciones únicas, terminadas y temporalmente determinadas. Los verbos (recibir, disparar y morir) que aparecen en la forma imperfectiva son télicos por su semántica, por lo que debieran ser usados en la forma perfectiva. A pesar de ello, el hablante, en este caso, el periodista, parece utilizar el pretérito imperfecto para no asumir completamente la responsabilidad de la información y remitirse a una fuente sin precisarla o a su propia 
conclusión. De este modo, se distancia de sus enunciados y atenúa al mismo tiempo el contenido de su proposición:

\begin{abstract}
(20) Al día siguiente de aparecer en las páginas de este diario un artículo suyo sobre la situación de la Universidad, el director del Colegio Mayor Diego de Covarrubias, Diego Mateo del Peral, recibía un oficio del rector de la Universidad Complutense de Madrid en el cual le comunicaba que había propuesto su cese inmediato a la junta de gobierno de la referida universidad. (El País, Educación, 04/08/1997)
\end{abstract}

(21) Un soldado croata disparaba ayer con antiaéreos contra posiciones terrestres serbias situadas a 200 metros. NIKOLA SOLIC. AP (La Vanguardia, Ciencia Militar, 02/05/1995)

(22) Hace algunos algunos días, con el título Cervantes y Shakespeare, salió publicada en su leído diario una erudita relación de la fecunda producción del famoso poeta inglés. Al final se afirma lo siguiente: «La más alta cumbre de las letras inglesas murió el 23 de abril de 1616. Ese mismo día moría el genio del idioma castellano con Miguel de Cervantes Saavedra». (El Tiempo, Literatura, 01/06/1990)

En otras lenguas románicas, como el francés, el portugués y el italiano, el uso del imperfecto para designar procesos acabados y para atenuar la responsabilidad del hablante es mucho menos prominente que en el español (cf. Böhm/Haßler/Mai en prensa), no obstante, se han podido encontrar algunos casos:

(23) fr. On y disait que le directeur du corps de ballet ne lui avait vraiment pas laissé de passedroit, la considérant comme un membre à part entière de sa troupe plutôt que comme une actrice. (source: $h$ ttp://www.voir.ca/cinema/fichefilm.aspx?ilDFilm=5045)

'Se decía que el director del cuerpo de baile verdaderamente no le había dado ningún privilegio, considerándola como un miembro separado de su tropa, más que como una actriz'.

(24) pt. Há já algum tempo alguém evidenciava num artigo do Jornal Terras da Beira, as qualidades da equipa candidata à Câmara Municipal da Guarda liderada por Carlos Andrade apelidando-a de "Dream Team» (CDP, 18 Set 97, A voz que vai morrer em directo.

'Hace algún tiempo alguien mostraba en un artículo del periódico Terras da Beira, las cualidades del equipo candidato para el ayuntamiento A Guarda, liderado por Carlos Andrade, apodándolo de «Equipo ideal»".

(25) it. Apollos Ivanov sembra aver trovato anche alleati potenti, scriveva ieri il giornale popolare russo Moskovski Komsomoliets, CORIS).

'Apollos Ivanov también parece haber encontrado aliados potentes, escribía ayer el periódico popular ruso Moskovski Komsommoliets'.

Sin embargo, es, sobre todo, llamativo el uso del imperfecto románico en informaciones bursátiles en las cuales se describen cifras y procesos concretos, pero los autores no quieren responsibilizarse de estos y se remiten a una fuente ajena sin mencionarla explícitamente:

(26) fr. L'action d'Isacsoft perdait 1,5 cent à 35,50 cents mardi après-midi à la Bourse de croissance TSX. (source: http://www.lapresseaffaires.com/article/20070327/LAINFORMER/70327146/5891/LAINFORMER01)

'La acción de Isacsoft perdía 1,5 centavos a 35,50 centavos el martes por la tarde en la bolsa de crecimiento TSX'.

(27) Repsol, que ayer pagaba dividendo movió 23.984 millones de pesetas, y Fecsa, que lo paga hoy 6.310 millones de pesetas. (El Mundo, 09/01/1996, Negocios)

(28) it. In avvio il colosso bancario newyorkese, che la scorsa settimana aveva perso oltre il $60 \%$ del suo valore, saliva infatti del 59\% a 6 dollari, WebCorp, II Sole 24 Ore, 24.11.2008).

Al inicio, el coloso bancario de Nueva York, que la semana pasada había perdido otro $60 \%$ de su 


\section{normas}

Intersección entre la evidencialidad y la atenuación: el pretérito imperfecto evidencial y el futuro narrativo | Gerda Haßler

valor, subía en efecto del 59\% a 6 dólares'.

Entre las lenguas románicas existen diferencias en el uso de las formas verbales para este propósito. En francés, esta función la asume, sobre todo, el Conditionnel journalistique (Raible 1983: 276; Dendale 2014), que no indica si se trata de evidencialidad inferencial o citativa, sino más bien que la información puede venir de una fuente o de la reflexión del periodista:

(29) fr. Les jeunes préféreraient que l'employeur se sente responsable de l'avenir qu'il leur prépare (2000-12-02)

'Los jóvenes preferirían que el empleador se sienta responsable por el futuro que les prepara'.

En otras lenguas, como el alemán, donde no hay formas imperfectivas, esta función es asumida por verbos modales que, además de su función deóntica, tienen usos evidenciales. En el ejemplo (30), la forma del verbo wollen marca la procedencia de una fuente, en este caso, de la persona que aparece como sujeto:

(30) al. Anders Behring Breivik hatte am Samstag die Täterschaft bei beiden Anschlägen zugegeben. Er will sie allein ausgeführt haben. (http://www.sueddeutsche.de/politik/bluttat-innorwegen-attentaeter-nennt-anschlaege-grausam-aber-notwendig-1.1123955)

'Anders Behring Breivik había admitido que fue el autor de ambos atentados ocurridos el sábado.

Él afirma haberlo realizado solo.'

Con el uso de will se establece una distancia del hablante frente al contenido de la proposición 'que él mismo ha ejecutado' y su contenido parece más bien improbable. Tambien con el verbo sollen que remite a una fuente, pero no se identifica como el sujeto, se atenúa el compromiso del hablante, pero se aproxima a la probabilidad:

(31) al. Behring Breivik soll ausdrücklich versichert haben, allein gehandelt zu haben. Doch stößt diese Darstellung bei den Behörden auf Skepsis. (http://www.stern.de/panorama/doppelanschlagin-norwegen-attentaeter-streitet-strafbare-handlung-ab-1709391.html)

'Behring Breivik aseguraba explícitamente haber actuado solo. Sin embargo, esta información ha sido recibida con escepticismo por las autoridades.'

El hecho de que en las lenguas románicas y, sobre todo, en español, sea el pretérito imperfecto que asuma la función de mitigación y de atenuación de la responsibilidad del hablante y de su contenido proposicional, a diferencia de que en alemán este papel es asumido por verbos modales, confirma la hipótesis de Abraham y Leiss que debido a la ausencia de verbos modales, el aspecto compensa esta falta de expresión: «In the absence of modal verbs, aspect fills the expressive gap» (Abraham/Leiss 2008: XIV).

\section{EL FUTURO NARRATIVO Y LA ATENUACIÓN}

Otra forma del indicativo que puede atenuar la fuerza ilocutiva, pero que es más infrecuente, en español, es el futuro narrativo. Su uso designa situaciones pasadas con respecto al momento de habla, pero también situaciones venideras desde un momento en el pasado, presupuesto por el hablante. Este uso aparece, sobre todo, en contextos narrativos, en los cuales puede utilizarse para designar una situación antes del momento de habla, pero después de un momento de referencia en el pasado. ${ }^{7}$

\footnotetext{
${ }^{7}$ Sobre estos usos del futuro, cf. Escandell (2010), Rodríguez Rosique (2008), García Negroni (2016).
} 


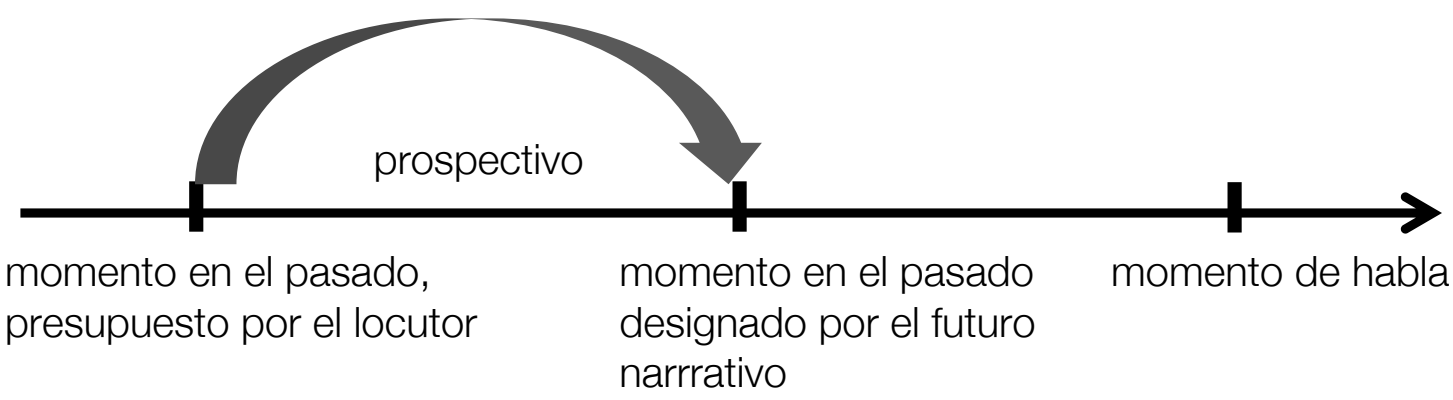

En español, este uso del futuro no se utiliza en el lenguaje cotidiano. Después de haber entrevistado a 20 hispanohablantes de España, Argentina, Colombia y Perú, todos los informantes especificaron nunca haberlo utilizado ni en el uso oral ni en el uso escrito. Su uso se encuentra, sin embargo, en textos narrativos e historiográficos, por medio del cual se relata situaciones pasadas que, desde un punto de referencia en el pasado, se consideran prospectivas:

(32) Lope de Vega nos ofrecerá en su dramaturgia un gran avance histórico.
(http://eljuego.free.fr/Fichas_gramatica/FG_futuro_anterior.htm\#22)

La realidad de la proposición [p = 'que Lope de Vega nos ofrece un gran avance histórico'] está mitigada por este uso del futuro. También en el siguiente ejemplo se trata de un futuro narrativo prospectivo y enciclopédico que describe la historia sobre el regreso de Hong Kong a la soberanía de China el 1 de julio de 1997. La historia es contada en el pasado, pero en forma prospectiva:

\begin{abstract}
(33) [...], empezaron a llegar grandes cantidades de refugiados desde Vietnam. C. El retorno a China con motivo de la proximidad del fin del arrendamiento británico sobre los Nuevos Territorios en el año 1997, dieron comienzo en 1982 una serie de conversaciones entre China y Gran Bretaña acerca del futuro de Hong Kong. El acuerdo legal entre estos dos países alcanzado en 1984 y firmado en el mes de diciembre en Pekín (la Declaración Conjunta chino - británica), estipulaba que todo Hong Kong retornaría a la soberanía china el 1 de julio de 1997. El territorio -que para entonces recibirá el nombre de Región Administrativa Especial China de Hong Kong- podrá mantener sus sistemas legal, social y económico durante, al menos, otros cincuenta años, y se les garantizará a sus ciudadanos las libertades civiles. China se hará cargo de la política exterior y la defensa del territorio. Después de 1997 Hong Kong tendrá, además, una constitución, conocida como Ley Básica, redactada por un comité convocado en Pekín, al que asistieron representantes de la colonia británica [...] (Corpus del Español, Enciclopedia: Hong Kong)
\end{abstract}

También el futuro compuesto puede utilizarse con significado prospectivo. Se establece un punto adicional en el pasado, antes del cual la situación tiene que ser terminada. Este punto temporal se encuentra después del momento en el pasado, presupuesto por el hablante, desde donde se inicia la prospección y llega hasta después del evento descrito en futuro compuesto:

(34) Y $\quad$ S se puede decir que la llegada del hombre a la luna en junio de 1969 habrá sido el primer paso de la conquista del espacio. (http://verbo.palabrita.net/Futuro-de-indicativo.html) 


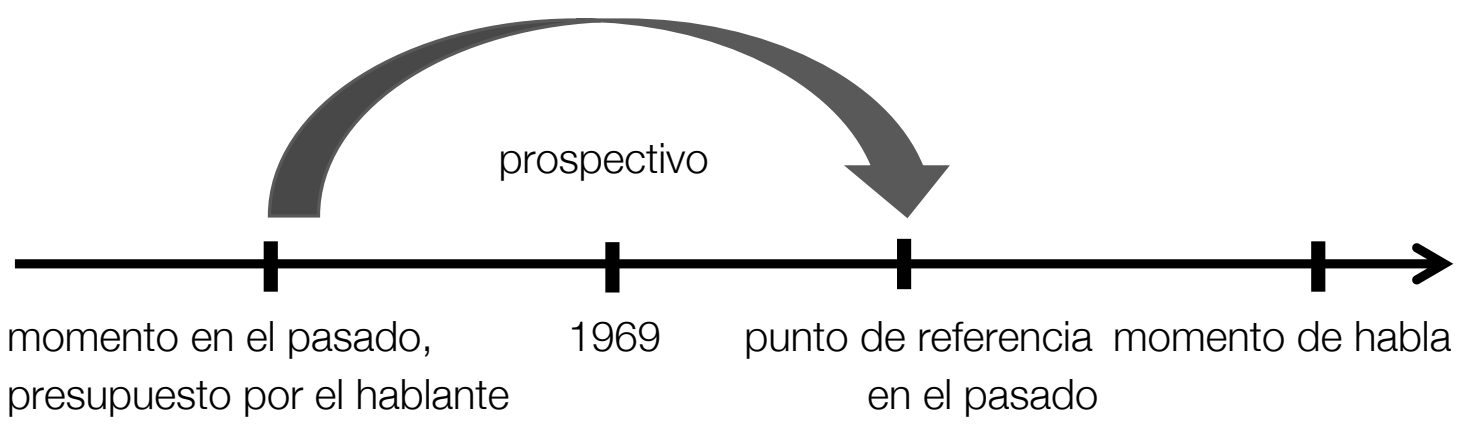

También se suele encontrar el uso del futuro compuesto con significado prospectivo en el pasado en obras literarias, donde se describe el drama de una narración en forma poética. En el siguiente ejemplo, el futuro compuesto es usado en alternancia con el presente narrativo para acercar al lector a la historia narrada:

\begin{abstract}
(35) Asústale el temor de que aquello dure indefinidamente; y comparándolos con tan grotesco cuadro, le parecen de color de rosa los que a él le echaron de su casa. Felizmente, no tarda la pupilera en anunciarle que desde el día siguiente comerá en su gabinete; porque para entonces habrá llegado la doncella que esperaba. Y como lo ofrece lo cumple. Gedeón come en su cuarto al otro día; y joh sorpresa embriagadora y confortativa! la doncella que ya vino, y le cubre la mesa, y después le sirve los manjares, es Solita; [...] (Corpus del Español, El buey suelto, De Pereda, José María, 1870)
\end{abstract}

En otras lenguas románicas, se utiliza el futuro narrativo más frecuentemente. Especialmente, en textos italianos, se presenta como un recurso usual de atenuación de afirmaciones que se introducen como proféticas desde un momento en el pasado, pero que ya se han cumplido antes del momento actual:

\footnotetext{
(36) it. Più tardi, Cosimo devrà capire che quando quel problema comune non c'è più, le associazioni non sono più buone come prima, e val meglio essere un uomo solo e non un capo. (l. Calvino, /l barone rampante)

'Más tarde, Cosimo deberá entender que cuando aquel problema común ya no exista, las asociaciones no son [serán] más buenas como antes, y vale mejor ser un hombre solo y no un jefe.'
}

En el siguiente ejemplo (37), el siglo XVI (Cinquecento) se introduce como un intervalo de referencia que precede a los siglos XVII y XVIII, en los cuales tenían lugar los acontecimientos, narrados en futuro simple (Bertinetto 1991: II, 117). La proposición sobre estos siglos subsiguientes se formula a partir de ese intervalo de referencia en el pasado:

(37) it. II Cinquecento ha distinto nettamente fra la civiltà e la primitività: fra il Seicento e il Settecento gli Scrittori distingueranno ulteriormente, nel senso della stessa civiltà [...] dando così maggior precisione di contorni al volto dell'Europa. (F. Chabod, Storia dell'idea d'Europa, Bari: Laterza 1961: 90)

'El siglo XVI ha distinguido claramente entre la civilización y la primitividad: entre el siglo XVII y XVIII los escritores distinguirán posteriormente en el sentido de la misma civilización [...]'. 


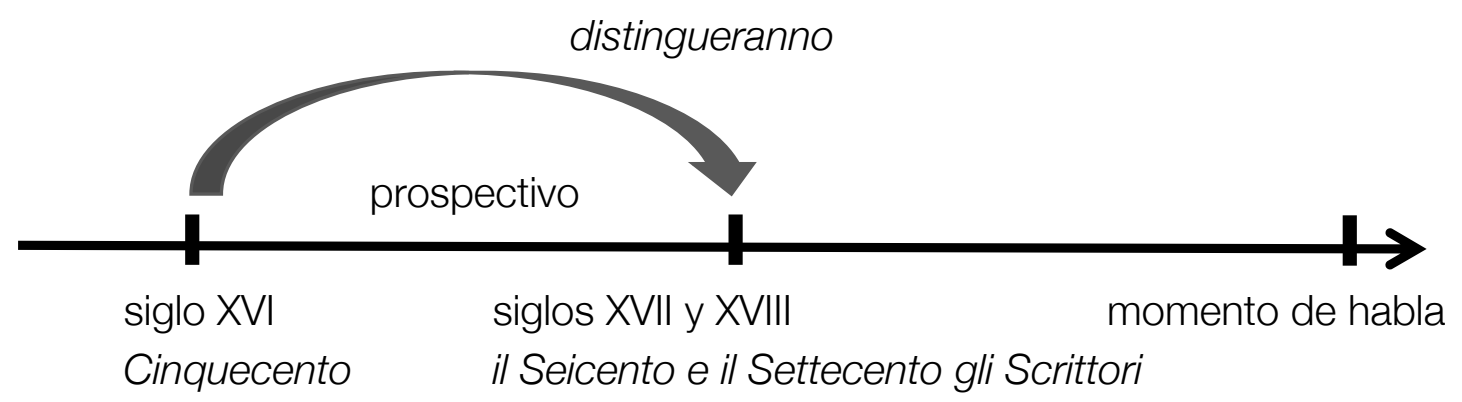

Para el francés, se ha encontrado en la gramática metódica de Riegel, Pellat y Rioul (1994: 313) una valoración ligeramente negativa del uso prospectivo del futuro anclado en un punto de referencia en el pasado. Al mismo tiempo, estos autores constatan su uso sobreabundante en composiciones escolares:

\footnotetext{
(38) fr. Victor Hugo naquit à Besançon en 1802. Ce fils d'un général d'Empire deviendra un des plus grands écrivains français.

'Victor Hugo nació en Besanzón en 1802. Este hijo de un general de imperio se convertirá en uno de los más grandes escritores franceses'.
}

Constatan también que tales usos del futuro en la prensa audio-visual representan acontecimientos históricos. Este uso del futuro en contextos del pasado, llamado por Riegel, Pellat y Rioul (1994: 313) futur d'anticipation, abre una perspectiva a las consecuencias de situaciones pasadas, pero las presenta atenuadas en su realidad.

Tales usos del futuro implican posterioridad, pero con referencia a un intervalo anclado en el pasado y mencionado en el contexto. Además, en estos casos se manifiestan coincidencias entre la modalidad y la temporalidad que provocan una atenuación de la asertividad de la proposición. El hablante finge con estos usos una dislocación retrospectiva con respecto al momento de habla. Por lo general, este futuro retrospectivo se utiliza solamente en la tercera persona. El carácter no-deíctico del futuro retrospectivo se comprueba también por su incompatibildad con adverbiales deícticos. Cuando el futuro se utiliza de una manera deíctica, no se puede interpretar como futuro retrospectivo, sino más bien como un futuro que expresa siempre posterioridad con respecto al momento de habla. (Bertinetto 1991: II, 117):

(39) it. II ragazzo partì all'improvviso. Tornerà solo dopo tre anni / *fra tre anni.

'El muchacho partió [se fue] de repente. Volverá después de tres años / *en tres años'.

También se puede atribuir al futuro narrativo un valor evidencial. El hablante se presenta en tales enunciados como alguien que conoce los hechos y comunica sus conclusiones. El hablante que narra los acontecimientos conoce la historia hasta su final. En consecuencia, puede deducir conclusiones de ciertas circunstancias y presentarlas con referencia a su propio acto cognitivo, pero que van ancladas a un futuro relativo al momento presupuesto por el hablante en el pasado. La proposición [p = 'que volverá después de tres años'] está mitigada en su validez por este uso evidencial del futuro.

Además del futuro, entre otros medios de realización de las funciones prospectiva y atenuadora, se destaca también el uso del verbo modal sollen en alemán:

(40) al. Im Januar 1932 entschloss er sich, London zu verlassen und nach Berlin zu gehen. Dies wird die folgenschwerste Entscheidung seines Lebens werden. 


\title{
normas
}

Intersección entre la evidencialidad y la atenuación: el pretérito imperfecto evidencial y el futuro narrativo | Gerda Haßler

\begin{abstract}
‘En enero 1932 decidió dejar Londres para irse a Berlín. Esta será la decisión trascendental en su vida'.

(41) al. Im Januar 1932 entschloss er sich, London zu verlassen und nach Berlin zu gehen. Dies sollte die folgenschwerste Entscheidung seines Lebens werden.

'En enero 1932 decidió dejar Londres para irse a Berlín. Esta deberá ser la decisión trascendental en su vida'.
\end{abstract}

\section{CONCLUSIÓN}

Los ejemplos del uso evidencial del pretérito imperfecto y del futuro narrativo prospectivo muestran que la marcación de la evidencialidad no se puede separar de la subjetivización del enunciado, de la relativización y de la perspectivización del hablante, así como de su expresión. La perspectivización del hablante resulta una categoría superordinada que incluye varias categorías solapantes (Hennemann 2013: 419):

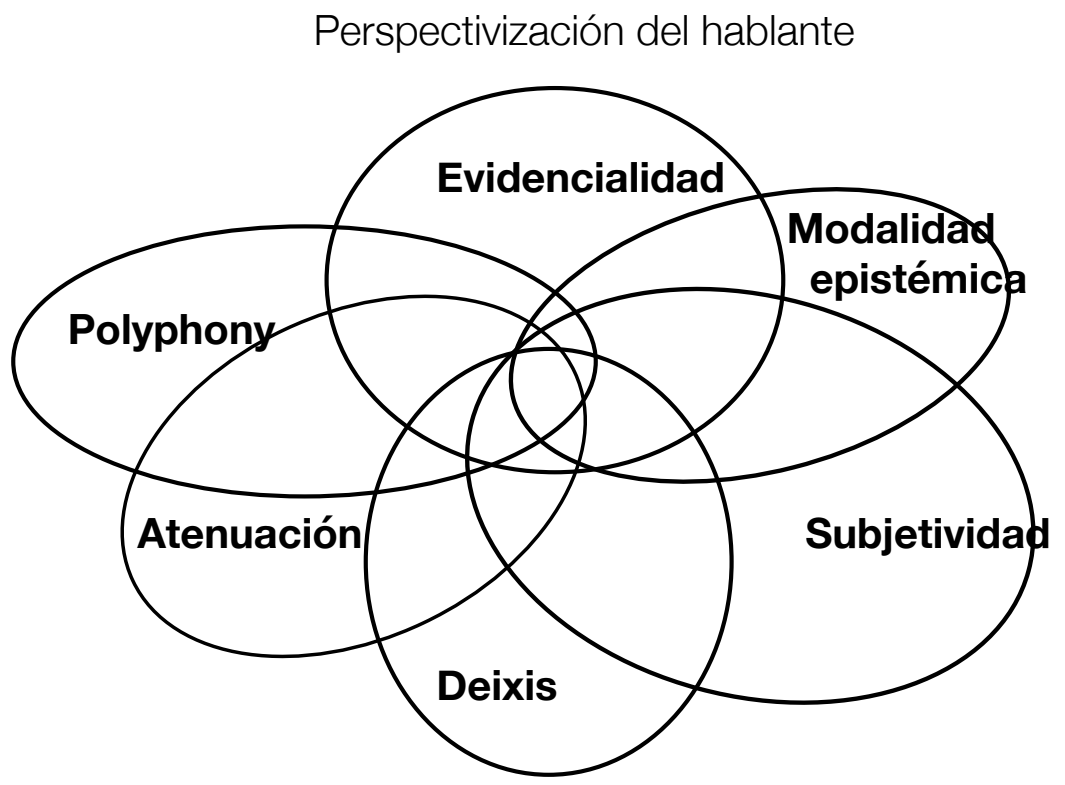

Las dos formas verbales descritas en esta contribución con significados secundarios, no prototípicos pueden atenuar la validez de la proposición y en consecuencia mitigar también la fuerza ilocutiva de los enunciados. En el caso del pretérito imperfecto, también se ha observado que se atenúa y se reduce la responsabilidad y el compromiso del hablante frente a su enunciado, ya que el hablante no menciona el autor original de la información, sino que tácitamente hace referencia a una fuente externa de información. De este modo, el distanciamiento del hablante de sus enunciados mediante el uso del pretérito imperfecto parece ser una estrategia pragmática-comunicativa de atenuación o mitigación, especialmente, en informaciones periodísticas, para no indicar necesariamente de dónde proviene la información y no asumir así completamente la responsabilidad de tales enunciados.

En las gramáticas, estos valores del préterito imperfecto y del futuro se exponen como «no rectos» y como excepciones, lo que se puede justificar por el uso relativamente poco frecuente de las formas verbales con estos significados. A este punto de vista se puede responder mediante dos reflexiones expuestas brevemente en este estudio: (1) Los significados no prototípicos de las formas verbales que permiten su uso como marcadores 
de la evidencialidad y de la mitigación son deducibles de sus rasgos temporales y aspectuales, lo que se ha mostrado en el ejemplo del puente metonímico de la aspectualidad hasta la modalidad, la evidencialidad y la atenuación. (2) El uso de las formas verbales discutidas con significados evidenciales y de atenuación surge por necesidades comunicativas, las que no solo son realizadas o expresadas por marcadores lexicales de atenuación, sino también por elementos del registro verbal.

\section{BIBLIOGRAFÍA}

ABRAHAM, Werner y Elisabeth LEISS, eds. (2008): Modality-Aspect Interfaces. Implications and typological solutions, Amsterdam, Philadelphia, John Benjamins Publishing Company.

BertinetTo, Pier Marco (1991): «ll verbo», en Renzi, Lorenzo, Giampaolo Salvi y Anna Cardinaletti, eds., Grande grammatica italiana di consultazione, vol. II: I sintagmi verbale, aggettivale, avverbiale. La subordinazione, Bologna, II Mulino, 13-161.

BeECHING, Kate (2002): Gender, Politeness and Pragmatic Particles in French. Amsterdam/Philadelphia: John Benjamins Publishing Company.

BöHM, Verónica (2013): «Evidentielle Markierung von fremden Äußerungen. Eine Analyse des spanischen Imperfekts in journalistischen Texten», en HENNEMANN, Anja y Claudia SCHLAAK, eds., Korpuslinguistische Untersuchungen. Analysen einzelsprachlicher Phänomene, Berlin, Frank y Timme GmbH, 119-130.

BöHM, Verónica (2016): La imperfectividad en la prensa española y su relación con las categorías semánticas de modalidad y evidencialidad, Frankfurt am Main, Peter Lang GmbH.

BöHM, Verónica y Anja HenNEMAnN (2014): «The Evidential Use of the Spanish Imperfect and the Conditional in Journalistic Contexts", Studia Neophilologica, 86, London, Routledge, Taylor y Francis Group, 1-18.

BöHM, Verónica, Gerda HABLER y Anja HENNEMANN (2017): «On the evidential use of English adverbials and their equivalents in Romance languages and Russian: A morpho-syntactic analysis», en Marín Arese, Juana Isabel et alii, Evidentiality Revisited. Cognitive grammar, functional and discourse-pragmatic perspectives, Amsterdam, Philadelphia: John Benjamins Publishing Company, 87-104.

BÖHM, Verónica, Gerda HABLER y Udo MAl (en prensa): "Relations entre l'aspectualité et la modalité dans les langues romanes étudiées à partir de textes journalistiques", en : Actes du XXVIII· Congresso internazionale di linguistica et filologia romanza. Roma

BRIZ, Antonio (1995): «La atenuación en la conversación coloquial. Una categoría pragmática», en CoRTÉs, Luis, ed., El español actual. Actas del I Simposio sobre análisis del discurso oral, Almería, Ediciones Universidad de Almería.

Briz, Antonio (1998): El español coloquial en la conversación. Esbozo de pragmagramática, Barcelona, Ariel Lingüística.

BRIZ, Antonio y Marta ALBELDA (2013): «Una propuesta teórica y metodológica para el análisis de la atenuación lingüística en español y portugués. La base de un proyecto en común (ES.POR.ATENUACIÓN)», Onomázein, 28, 288 - 319

CORNILLIE, Bert (2007): Evidentiality and Epistemic Modality in Spanish (Semi-)Auxiliaries. A Cognitive-Functional Approach, Berlin, Mouton de Gruyter.

CORPUS DEL ESPAÑOL: http://www.corpusdelespanol.org/.

DENDALE, Patrick (2014): «Le conditionnel de reprise: apparition en français et traitement dans les grammaires du XVle et au XXe siecle», en Anscombre, Jean-Claude, Evelyne Oppermann-Marsaux y Amalia Rodríguez Somolinos, Médiativité, polyphonie et modalité en français.Études synchroniques et diachroniques, Paris, Presses Sorbonne Nouvelle, 19-34.

DESsì SCHMID, Sarah (2014): Aspektualität. Ein onomasiologisches Modell am Beispiel der romanischen Sprachen, Berlin/Boston, De Gruyter.

DíAz SAlgado, Luis C. (2000): «La Decoración del Drama», Venezuela Analítica 2000, Núm. NOV 2000, 1-1. http://www.buenastareas.com/ensayos/La-Decoraci\%C3\%B3n- Del-Drama/3864447.html (30/04/2015).

DieWALD, Gabriele (1999): Die Modalverben im Deutschen. Grammatikalisierung und Polyfunktionalität, Tübingen, Max Niemeyer Verlag.

DieWALD, Gabriele y Elena SmIRNOVA (2010): Evidentiality in German. Linguistic Realization and Regularities in Grammaticalization, Berlin, Mouton de Gruyter.

EsCANDELL, Mª Victoria (2010): «Futuro y evidencialidad», Anuario de Lingüística Hispánica, 26, 9-34.

GARCÍA FERNÁNDEZ, Luis (2004): «El pretérito imperfecto: Repaso histórico y bibliográfico», en GARCÍA FERNÁNDEZ, Luis y Bruno CAMUS BeRGARECHE, eds., El pretérito imperfecto, Madrid, Gredos, 13-95. 


\section{normas}

Intersección entre la evidencialidad y la atenuación: el pretérito imperfecto evidencial y el futuro narrativo | Gerda Haßler

García NeGronI, María Marta (2016): «Polifonía, evidencialidad citativa y tiempos verbales. Acerca de los usos citativos del futuro morfológico y del futuro perifrástico», en González Ruiz, Ramon, Izquierdo Alegría, Dámaso y Loureda, Óscar, La evidencialidad en español: teoría y descripción, Iberoamericana/Vervuert.

GutiÉRREZ ARÁUS, María Luz (1995): «Sobre los valores secundarios del imperfecto», en Rueda, M. et alii, Actuales tendencias en la enseñanza del español como lengua extranjera II. Actas del VI congreso internacional de ASELE (León 5-7 de octubre 1995), Actas VI, León, Universidad de León, 177-186.

HABLER, Gerda (2010): «Epistemic modality and evidentiality and their determination on a deictic basis», en Becker, Martin G., Eva-Maria Remberger, eds., Modality and Mood in Romance. Modal Interpretation, Mood Selection, and Mood Alternation, Berlin, De Gruyter, 95-108.

HABLER, Gerda (2012): «Indicative Verb Forms as Means of Expressing Modality in Romance Languages», en Abraham, Werner, Elisabeth Leiss, Covert Patterns of Modality, Cambridge, Cambridge Scholars, 133-152.

HAßLER, Gerda (2015): «Evidentiality and the expression of speaker's stance in Romance languages and German», $\quad$ Discourse $\quad$ Studies, $\quad 2015, \quad 182 \quad 1(2), \quad 209$. http://dis.sagepub.com/content/17/2/182.full.pdf?ijkey=cAuNmS6BMqjbk7p\&keytype=finite DOI: 10.1177/1461445614564522.

HABLER, Gerda (2016): Temporalität, Aspektualität und Modalität in romanischen Sprachen, Berlin, Boston, De Gruyter.

Hennemann, Anja (2013), A Context-Sensitive and Functional Approach to Evidentiality in Spanish or Why Evidentiality needs a Superordinate Category, Frankfurt am Main, Peter Lang GmbH.

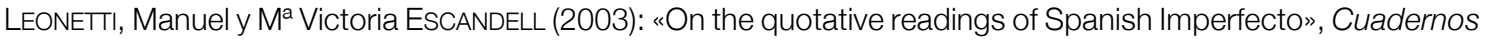
de Lingüística, 10, 135-154.

RAIBLE, Wolfgang (1983): "Knowing and Believing - and Syntax», en Parret, Herman, On Believing. Epistemological and Semiotic Approaches - De la croyance. Approches épistémologiques et sémiotiques, Berlin, De Gruyter, 275-291

REAL ACADEMIA ESPAÑOLa (2009): Nueva gramática de la lengua española (NGLE), Madrid, Espasa Libros.

REYES, Graciela (1990): «Tiempo, modo, aspecto e intertextualidad», Revista Espanola Lingüística, 1(20), 1753.

REYES, Graciela (1994): Los procedimientos de cita: citas encubiertas y ecos, Madrid, Arco Libros.

RIEGEL, Martin, Jean-Christophe PeLLAT y René RIOUL (1994): Grammaire méthodique du français, Paris, Presses Universitaires de France.

RodRíGUEZ Rosique, Susana (2008): «Las ventanas de la gramática: discurso y futuro distanciador», Círculo de Lingüística Aplicada a la Comunicación, 55, 111-132.

SQUARTINI, Mario (2001): «The internal structure of evidentiality in Romance», Studies in Language, 25/2, 297334.

VolKMANn, Gesina (2005): Weltsicht und Sprache. Epistemische Relativierung am Beispiel des Spanischen, Tübingen, Gunter Narr. 